Supporting Information

\title{
Infrared Laser Activation of Soluble and Membrane Protein Assemblies in the Gas Phase
}

Victor A. Mikhailov, Idlir Liko, Todd H. Mize, Matthew F. Bush, Justin L. P. Benesch and Carol V. Robinson*

Department of Chemistry, Physical and Theoretical Chemistry Laboratory, University of Oxford, Oxford, OX1 3QZ, United Kingdom

\# Present address: Department of Chemistry, University of Washington, Box 351700, Seattle, WA 98195-1700

*Corresponding Author: carol.robinson@chem.ox.ac.uk

\section{Contents}

1. Table S1. Parameters of IR laser radiation used for activation of protein complexes.

2. Figure S1. Laser beam fluence along the axis of the ion trap.

3. Note S1. Estimation of the average time between collisions in the ion trap.

4. Figure S2. Dissociation mass spectra of avidin tetramer and octamer.

5. Figure S3. Continuous IRMPD of avidin at different bath gas pressures.

6. Figure S4. IRMPD and CID mass spectra of $\mathrm{CS}_{2}$ hydrolase.

7. Figure S5. IRMPD mass spectra of glutamate dehydrogenase hexamer and dodecamer.

8. Figure S6. Mass spectra of membrane proteins before and after IR activation. 


\begin{tabular}{|c|c|c|c|c|}
\hline IR pulse duration /wavelength & $\begin{array}{c}\text { continuous } / 10.6 \\
\mu \mathrm{m}(\mathrm{LW}-\mathrm{IR})\end{array}$ & $\begin{array}{l}100 \mathrm{~ns} / \\
10.6 \mu \mathrm{m} \\
(\mathrm{LW}-\mathrm{IR})\end{array}$ & $\begin{array}{c}60 \mu \mathrm{s} / \\
2.94 \mu \mathrm{m} \\
(\mathrm{MW}-\mathrm{IR})\end{array}$ & $\begin{array}{l}50 \mathrm{~ns} / \\
2.94 \mu \mathrm{m} \\
(\mathrm{MW}-\mathrm{IR})\end{array}$ \\
\hline $\begin{array}{l}\text { Maximum IR energy }{ }^{(1)} \\
\text { at entrance to MS, } \boldsymbol{E}_{\text {in }}\end{array}$ & $5.6 \mathrm{~J}$ & $0.55 \mathrm{~J}$ & $40 \mathrm{~mJ}$ & $15 \mathrm{~mJ}$ \\
\hline Trapping Time, $\boldsymbol{t}$ & $243 \mathrm{~ms}$ & $93 \mathrm{~ms}$ & $493 \mathrm{~ms}$ & $493 \mathrm{~ms}$ \\
\hline $\begin{array}{l}\text { Maximum IR Power }{ }^{(2)} \text { at } \\
\text { entrance to MS, } \boldsymbol{P}_{\text {in }}\end{array}$ & $23.2 \mathrm{~W}$ & $5.5 \mathrm{MW}$ & $670 \mathrm{~W}$ & $0.3 \mathrm{MW}$ \\
\hline $\begin{array}{l}\text { IR energy at exit from MS }{ }^{(3)} \text {, } \\
\boldsymbol{E}_{\text {out }} \text { as } \% \text { of } \boldsymbol{E}_{\text {in }}\end{array}$ & $50 \%$ & $20 \%$ & ca. $100 \%$ & ca. $100 \%$ \\
\hline $\begin{array}{l}\text { Laser beam diameter at the } \\
\text { laser output mirror }\end{array}$ & $3.5 \mathrm{~mm}$ & $11.6 \mathrm{~mm}$ & $3 \mathrm{~mm}$ & $3 \mathrm{~mm}$ \\
\hline Laser beam divergence & $4 \mathrm{mrad}$ & $4 \mathrm{mrad}$ & $<1 \mathrm{mrad}$ & $<1 \mathrm{mrad}$ \\
\hline Beam propagation factor, $\boldsymbol{M}^{2}$ & 1.2 & 3.29 & 1 & 1 \\
\hline $\begin{array}{l}\text { Focused beam dimeter at the } \\
\text { beam entrance to ion trap }\end{array}$ & $1.03 \mathrm{~mm}$ & $1.91 \mathrm{~mm}$ & $0.61 \mathrm{~mm}$ & $0.61 \mathrm{~mm}$ \\
\hline $\begin{array}{l}\text { Laser fluence at the beam } \\
\text { entrance to ion trap }\end{array}$ & $670 \mathrm{~J} / \mathrm{cm}^{2}$ & $19.2 \mathrm{~J} / \mathrm{cm}^{2}$ & $13.7 \mathrm{~J} / \mathrm{cm}^{2}$ & $5.1 \mathrm{~J} / \mathrm{cm}^{2}$ \\
\hline Radiation bandwidth ${ }^{(4)}$ & $\begin{array}{c}5.4 \mathrm{~cm}^{-1}(10.57- \\
10.63 \mu \mathrm{m})\end{array}$ & $<0.13 \mathrm{~cm}^{-1}$ & $\sim 1-3 \mathrm{~cm}^{-1}$ & $\sim 1-3 \mathrm{~cm}^{-1}$ \\
\hline
\end{tabular}

Table S1. Parameters of IR laser radiation used for activation of protein complexes.

Laser pulse energy / power was measured before $\left(\boldsymbol{E}_{\text {in }}, \boldsymbol{P}_{\text {in }}\right)$ and after the ion trap $\left(\boldsymbol{E}_{\text {out }}, \boldsymbol{P}_{\text {out }}\right)$ to estimate possible radiation losses on the apertures of the MS elements (2-3 mm diameter) due to the different beam sizes and divergences from different lasers used in the experiments. $\boldsymbol{E}_{\text {in }}$ and $\boldsymbol{P}_{\text {in }}$ were measured at the top entrance IR mirror, and $\boldsymbol{E}_{\text {out }}$ and $\boldsymbol{P}_{\text {out }}$ were measured with the ion guide removed and the power meter put in its place, Figure 1A in manuscript. The energy of the radiation inside the ion trap, $\boldsymbol{E}_{\text {trap }}$ was in between those two values, $\boldsymbol{E}_{\text {out }}<\boldsymbol{E}_{\text {trap }}<\boldsymbol{E}_{\text {in. }}$. The largest losses were induced for the pulsed $\mathrm{CO}_{2}$ laser because of its largest beam aperture $(11.6 \mathrm{~mm})$ and 
propagation factor $\left(\boldsymbol{M}^{2}=3.29\right)$. The smallest, almost negligible losses were induced for the $2.94 \mu \mathrm{m}$ Er:YAG laser beam (beam diameter $=3 \mathrm{~mm}$, divergence $<1 \mathrm{mR}$ ). Laser beam parameters at laser output were taken from laser specifications by manufacturers, and used to calculate the beam parameters in the ion trap (1830 mm free beam path followed by $500 \mathrm{~mm}$ focusing by a lens).

${ }^{(1)}$ For pulsed radiation $\boldsymbol{E}_{\text {in }}$ is the energy of the single laser pulse. For continuous radiation: $\boldsymbol{E}_{\boldsymbol{i n}}=$ $\boldsymbol{P}_{\text {in }} \times \boldsymbol{t}$, where $\boldsymbol{t}$ is the trapping time.

${ }^{(2)}$ Maximum IR power is the maximum output power of the continuous laser or, in the case of a pulsed laser, the estimated average power of the laser pulse: $\boldsymbol{P}=\boldsymbol{E}_{0} / \tau$, where $\tau$ is the pulse duration.

${ }^{(3)}$ Measured in the ion guide region when the mass spectrometer was vented and open.

(4) Bandwidth values for the $\mathrm{CO}_{2}$ lasers are provided from their specifications, bandwidth value for the Er:YAG laser are quoted as typical from the literature. 


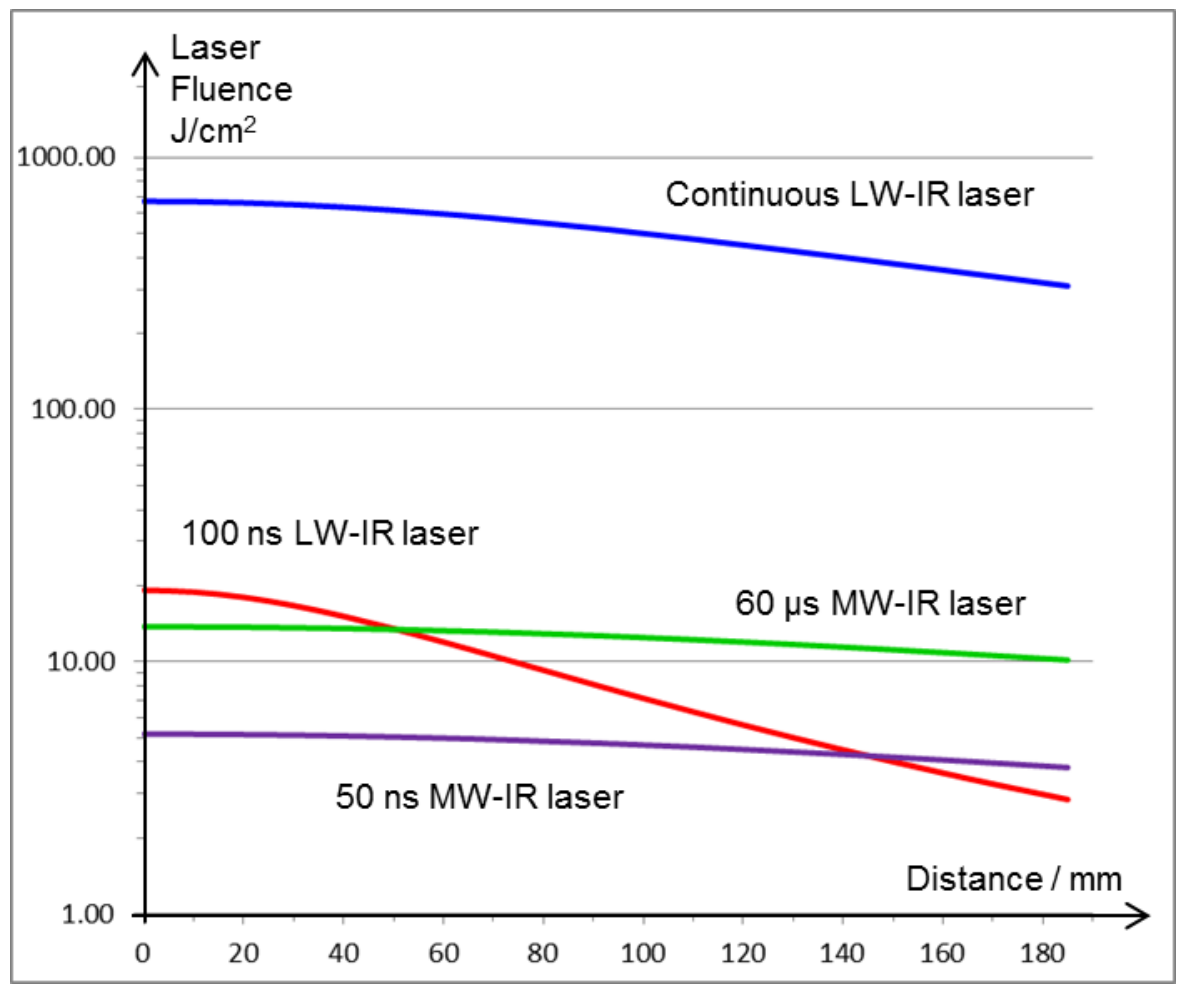

Figure S1. Laser beam fluence along the axis of the ion trap. Variations in the laser beam fluence (=laser radiation energy/laser beam area) are due to the beam expansion in the ion trap (trap length=185 mm) after it was focused at the trap exit electrode (Distance=0). Beam areas are calculated for the $500 \mathrm{~mm}$ focusing lens, and using beam parameters from the laser specifications (beam diameter, divergence and propagation factor $\mathrm{M}^{2}$, Table S1). Note, that all three pulsed irradiation regimes (one for the pulsed LW-IR laser and two for the pulsed MW-IR laser) have much lower laser fluence than the continuous 100-ns LW-IR regime along all the length of the ion trap. The 100-ns LW-IR laser demonstrates the largest decrease in fluence with the distance in the trap because of its largest output beam diameter $(11.6 \mathrm{~mm})$ and beam propagation factor $\left(\mathrm{M}^{2}=3.29\right)$ among the three IR lasers used in the experiments. 


\section{Note S1. Estimation of the average time between collisions in the ion trap.}

The mean free path of an ion in the trap: $\boldsymbol{L}=(\sqrt{2} \sigma n)^{-1}$, where $\sigma$ is the ion cross section and $\boldsymbol{n}$ is the number density of the collision gas in the cell. For trapping of avidin (64 kDa) the pressure in the trap is $\approx 10^{-4}$ mbar. Number density of the ideal gas at $10^{-4}$ mbar pressure is $\boldsymbol{n} \approx 10^{-7} \boldsymbol{n}_{\boldsymbol{a t m}}=$ $2.504 \times 10^{18} \mathrm{~m}^{-3}$, where $\boldsymbol{n}_{\boldsymbol{a t m}}=2.504 \times 10^{25} \mathrm{~m}^{-3}$ is the gas number density at the atmospheric pressure. Collision cross section of avidin ions (charge states $15+$ to $17+$ ) in nitrogen is ca. $41.5 \mathrm{~nm}^{2}$ (Bush et al. Anal. Chem. 2010, 82, 9557: Supporting Information). The mean free path for avidin ions is: $\boldsymbol{L}=6.77 \times 10^{-3} \mathrm{~m}=6.77 \mathrm{~mm}$. Root-mean-square speed of the gas molecules in the ion trap is: $\boldsymbol{v}$ $=\left(3 \boldsymbol{R} T / \boldsymbol{M}_{\boldsymbol{m}}\right)^{1 / 2}$, where $\boldsymbol{R}$ is the molar gas constant, $\boldsymbol{T}$ is the temperature (293 K) and $\boldsymbol{M}_{\boldsymbol{m}}$ is the molar mass $(\mathrm{kg} / \mathrm{mol})$. For nitrogen $\boldsymbol{v}=511 \mathrm{~m} / \mathrm{s}$. Therefore, the average time between collisions is: $\boldsymbol{t}=\boldsymbol{L} / \boldsymbol{v}=1.32 \times 10^{-5} \mathrm{~s}=13.2 \mu \mathrm{s}$.

The collision cross section of GroEL (803 $\mathrm{kDa}$ ) is larger, in the range of $219-244 \mathrm{~nm}^{2}$ (Bush et al. Anal. Chem. 2010, 82, 9557, van Duijn et al., J. Am. Chem. Soc., 2009, 131, 1452), and the trap pressure was higher (about $10^{-3} \mathrm{mbar}$ ): thus the average time between collisions is much shorter, са. $0.23 \mu$ s. For slightly heavier collision gases, e.g. Ar, the time changes insignificantly, and the difference originates mostly from the difference in the root-mean-square speed of the gas particles, i.e. proportionally to the factor of $\left(\mathrm{M}_{\mathrm{Ar}} / \mathrm{M}_{\mathrm{N} 2}\right)^{1 / 2}=1.20$. That gives the average time between collisions values of 15.7 and $0.28 \mu$ s for avidin and GroEL, respectively. Note that in both cases of avidin and GroEL, the average time between collisions is longer than the 50- and 100-ns laser pulses that we used for IR activation in some of the experiments. 

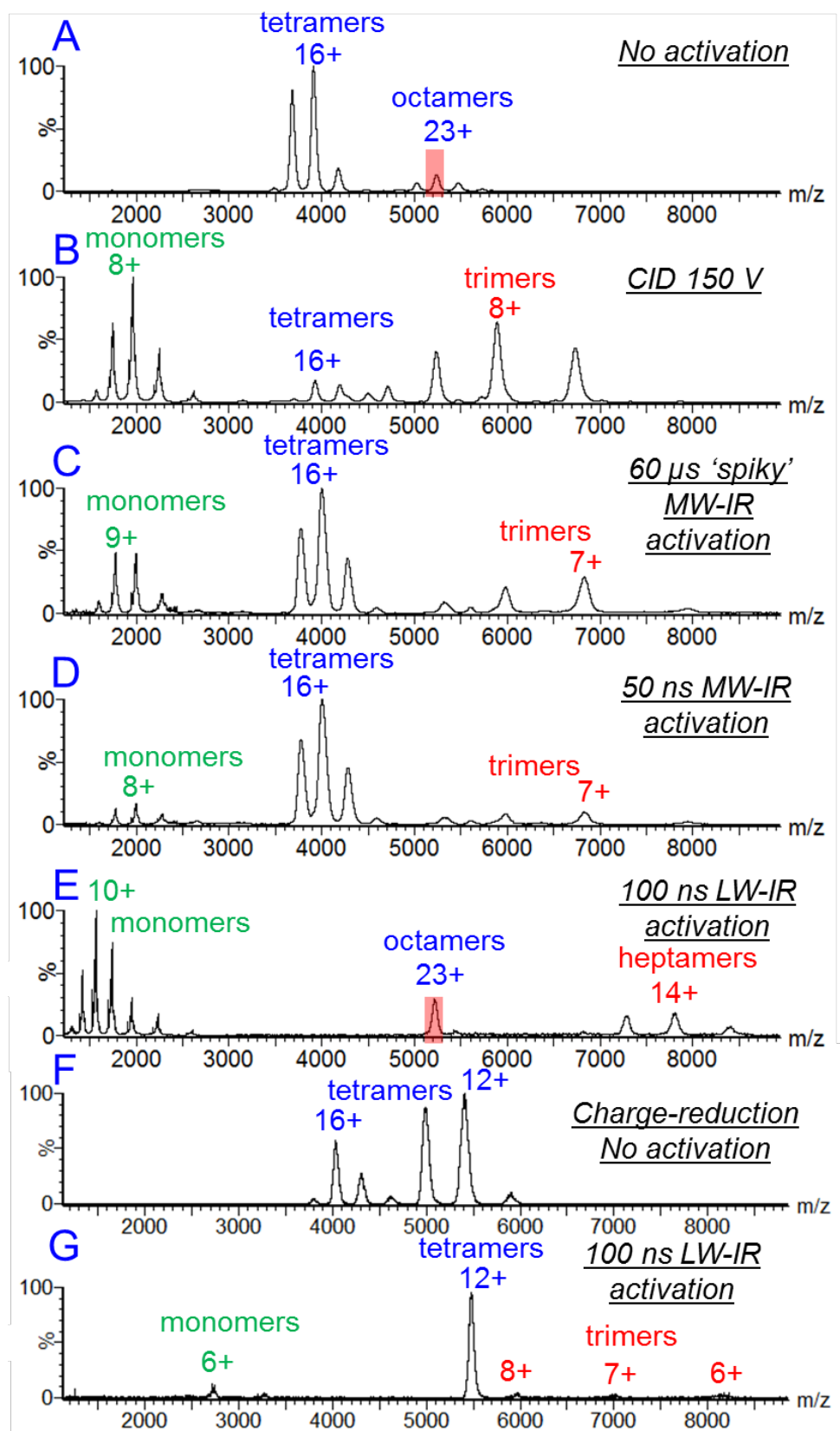

Figure S2. Mass spectra of avidin. A) Mass spectrum of avidin from concentrated $30 \mu \mathrm{M}$ solution in $200 \mathrm{mM}$ ammonium acetate. Octamers (tetramer self-adduct) are present. B) Mass spectrum of avidin after CID with a collision voltage $150 \mathrm{~V}$ (less concentrated solution at $5 \mu \mathrm{M}$ ). C) Mass spectrum of avidin (5 $\mu \mathrm{M})$ after pulsed MW-IR (2.94 $\mu \mathrm{m})$ activation by 'spiky' 60- $\mu$ s laser pulses (fragment yield 45.3\%). D) Mass spectrum of avidin (5 $\mu \mathrm{M}$ ) after activation by 50-ns MW-IR laser 
pulses (fragment yield 25.0\%). E) IRMPD mass spectrum of mass-selected 23+ charge state of avidin octamer (30 $\mu \mathrm{M})$, pulsed 100-ns LW-IR activation used and asymmetric dissociation observed. F) Mass spectruma of avidin from solution with $5 \mathrm{mM}$ of triethylamine acetate (TEA) leading to the appearance of charge-reduced tetramer ions $(13+, 12+$ and $11+)$. G) IRMPD of the 12+ charge-reduced tetramer ions with $100 \mathrm{~ns}$ LW-IR pulses results in asymmetric dissociation. 

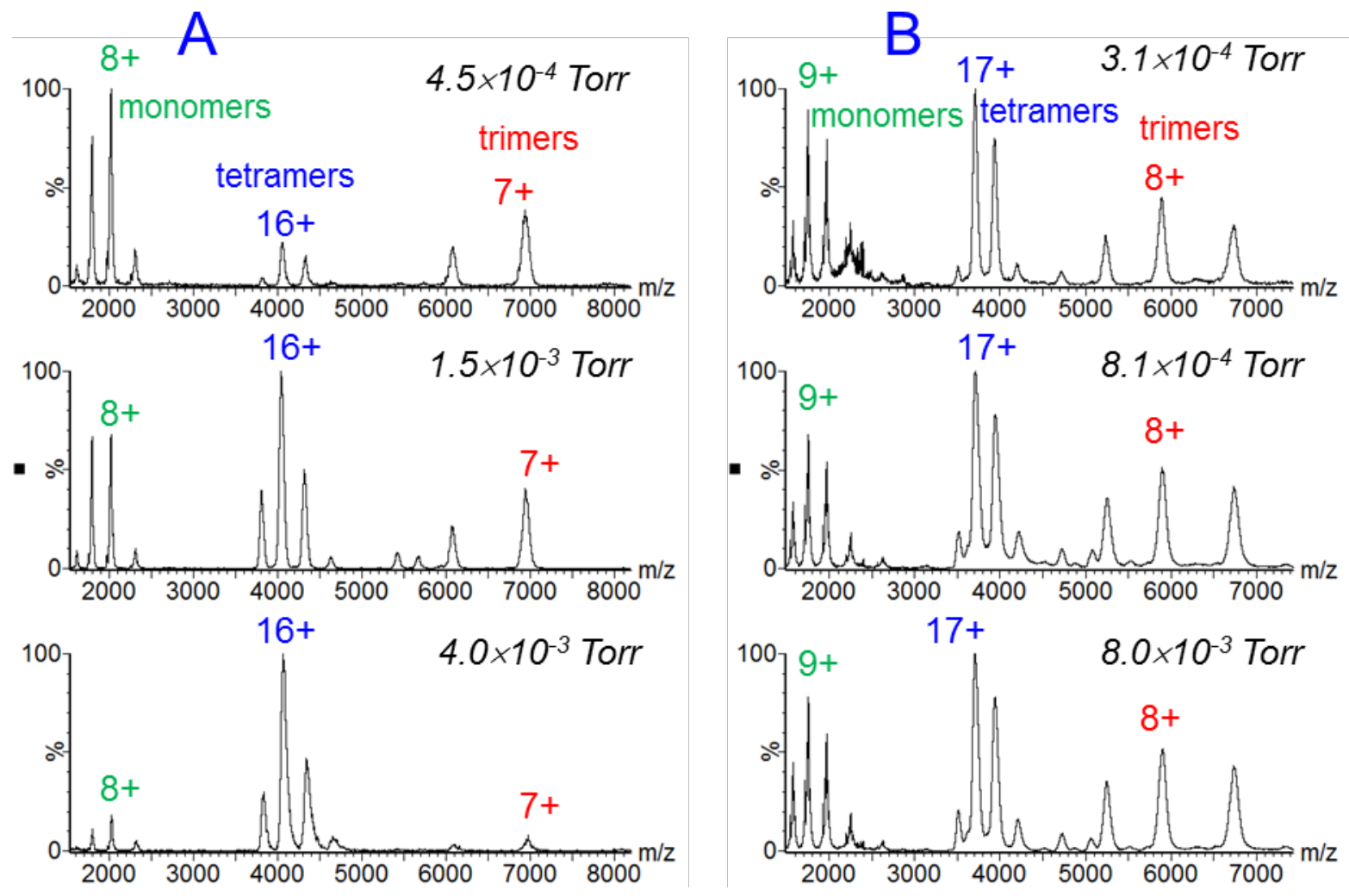

Figure S3. Continuous and pulsed IRMPD of avidin at different bath gas (argon) pressures in the ion trap. A) Continuous IRMPD at 95\% laser power, 243 ms trapping time: dissociation efficiency decreases with the pressure increasing from $4.5 \times 10^{-4}$ to $4 \times 10^{-3} \mathrm{mbar}$, as evidenced by the decreasing abundances of fragment monomers and trimers. B) 100-ns LW-IRMPD at $0.2 \mathrm{~J}$ pulse energy. The dissociation efficiency (yield of fragment monomers and trimers) remains almost constant, ca. $60 \%$ in the range of trap pressure from $3.1 \times 10^{-4}$ to $8 \times 10^{-3}$ mbar. 


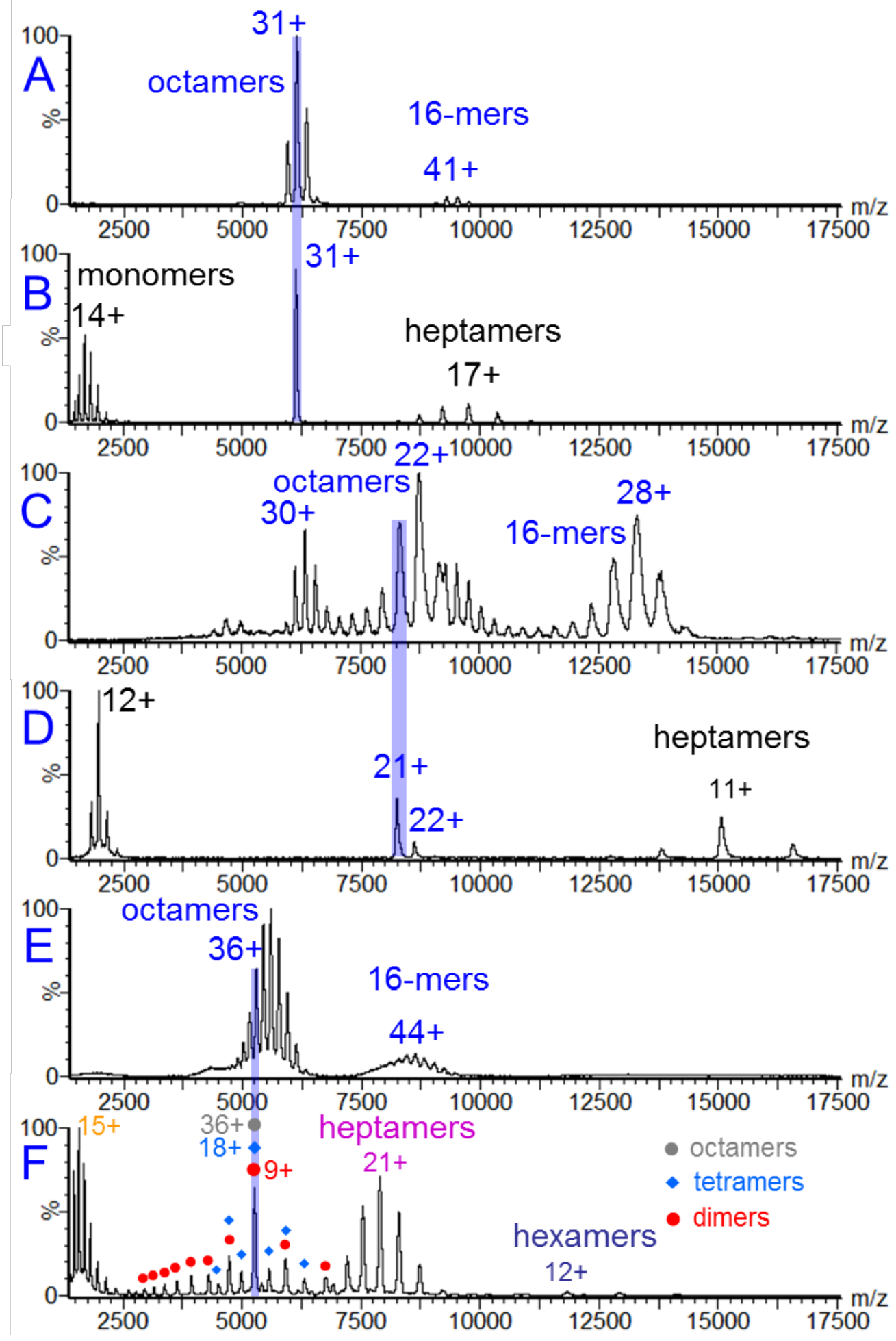


Figure S4. Mass spectra of $\mathrm{CS}_{2}$ hydrolase. A) Mass spectrum of the hydrolase octamer and hexadecamer from $200 \mathrm{mM}$ ammonium acetate solution. B) IRMPD of 31+ charge state of hydrolase octamer with 100-ns LW-IR laser pulses. C) Mass spectrum of charge-reduced ions of the hydrolase from solution with $5 \mathrm{mM}$ triethylamine acetate (TEA). D) IRMPD of charge-reduced 21+ charge state of the hydrolase octamer with 100-ns LW-IR pulses. E) Mass spectrum of the hydrolase from the solution with $5 \%$ sulfolane. F) CID (100 V collision voltage) mass spectrum of the mass-selected 36+ charge state of the octamer. Both symmetric fragments (dimers and tetramers) and asymmetric fragments (monomers and heptamers) are observed. 


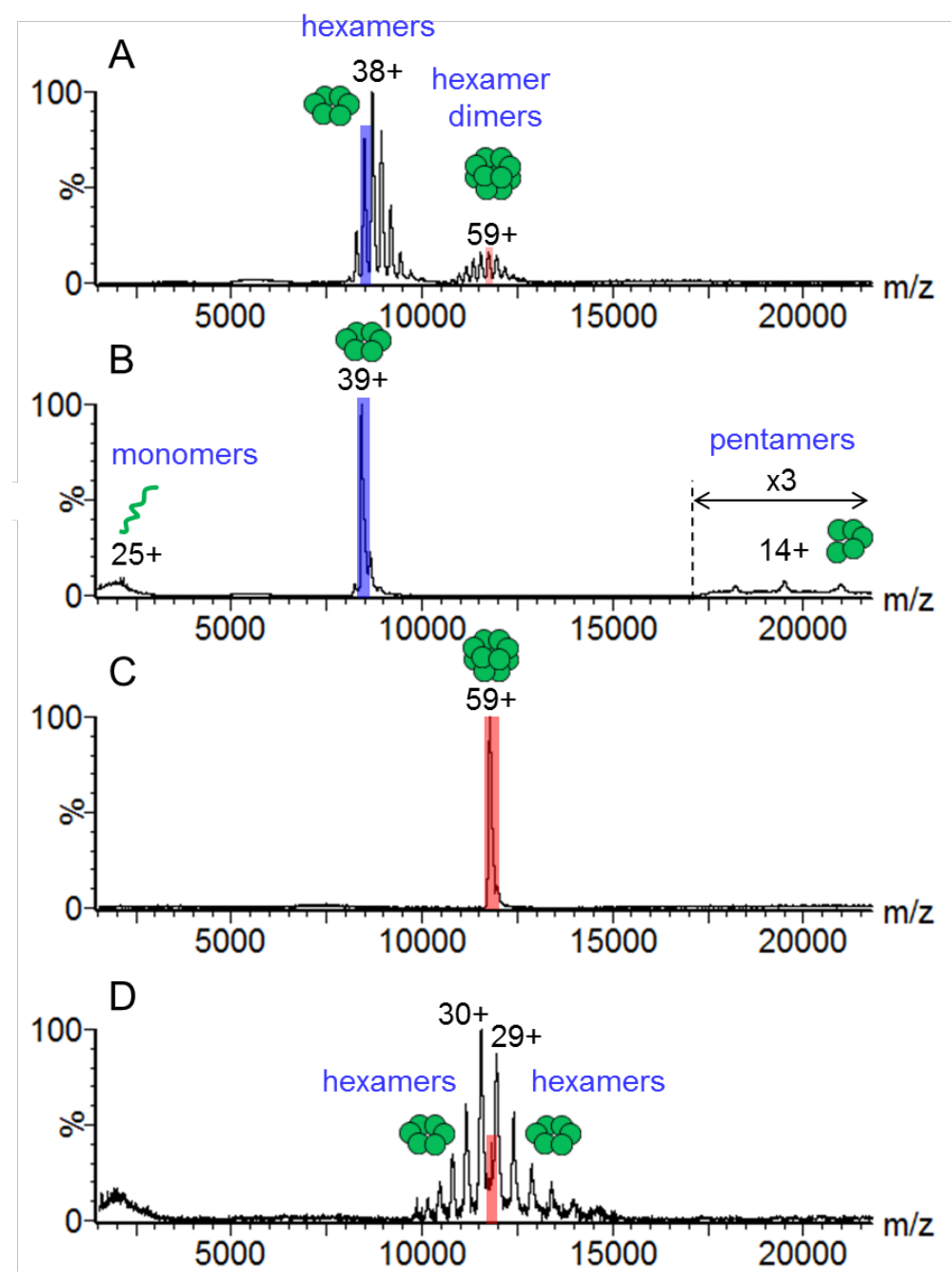

Figure S5. Mass spectra of glutamate dehydrogenase (GD). (A) No activation. (B) 100 ns / 10.6 $\mu \mathrm{m}$ IRMPD of the 39+ charge state of the GD hexamer: very weak asymmetric dissociation observed. (C) Mass selection of the 59+ charge state of the GD dodecamer (hexamer dimer). (D) $100 \mathrm{~ns} / 10.6 \mu \mathrm{m}$ IRMPD of the 59+ charge state of the dodecamer results in symmetric dissociation into the hexamers. 


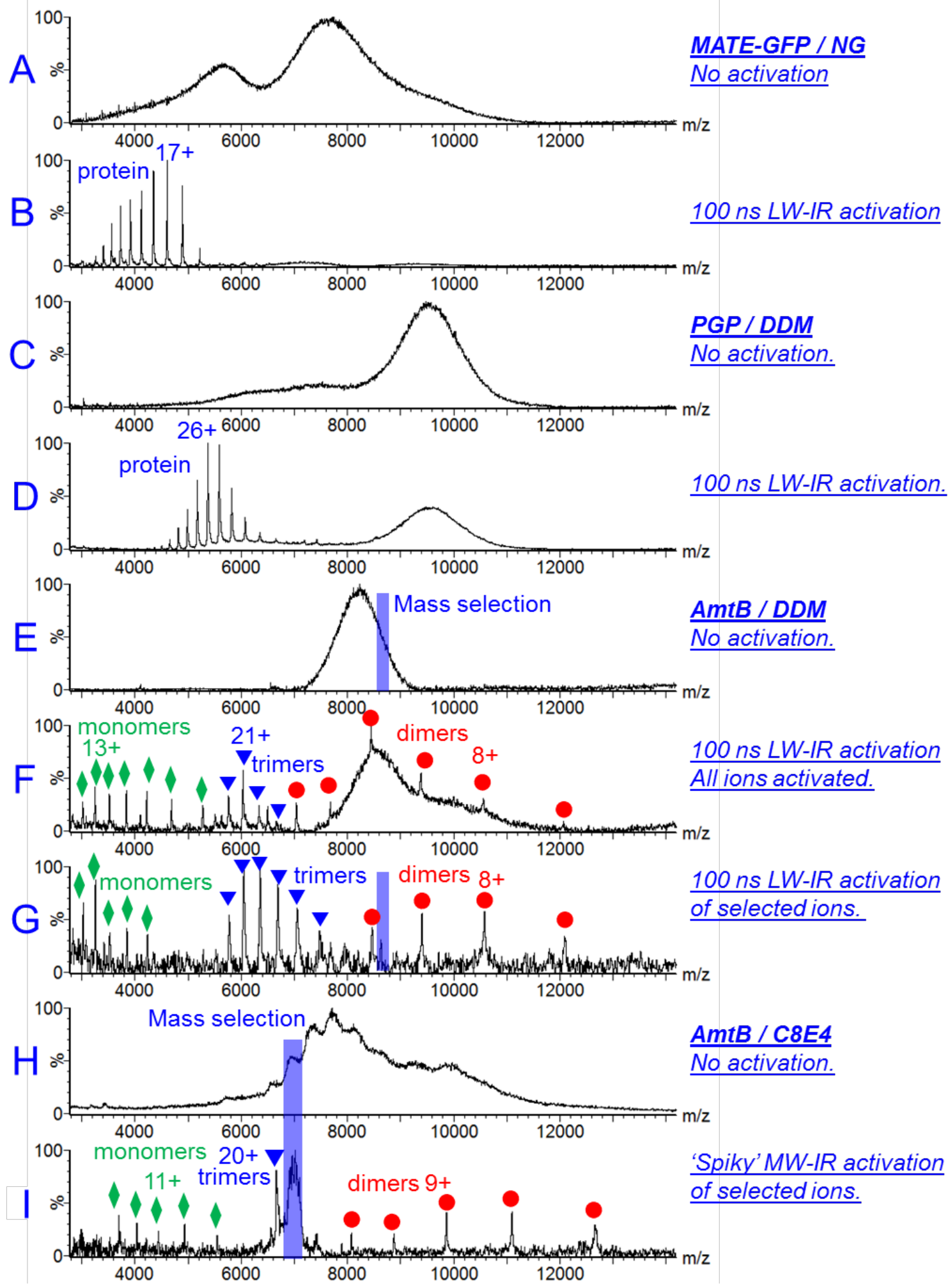


Figure S6. Mass spectra of membrane proteins before and after IR activation. (A) Fused MATEGFP membrane protein in NG micelles without activation (MATE - multi-antimicrobial extrusion membrane protein, GFP - green fluorescent protein). (B) Same protein after 100-ns LW-IR (10.6 $\mu \mathrm{m}$ ) activation. (C) P-glycoprotein in DDM micelles (no activation), and (D) after $100 \mathrm{~ns}$ LW-IR activation. (E) AmtB trimer in DDM micelles (no activation), (F) after 100-ns LW-IR activation of all ions and (G) after 100-ns LW-IR activation of the mass selected part of the ion signal (indicated by a blue band). (H) AmtB trimer from different species in C8E4 micelles and (I) after 60 - $\mu \mathrm{s}$ MW-IR $(2.94 \mu \mathrm{m})$ activation of the mass selected part of the ion signal. 